\title{
Plebiscito en el cantón de Río Cuarto: Un ejercicio sin precedentes de democracia directa en Costa Rica
}

Recibido: 4 de mayo 2020 Revisado: 3 de marzo 2020 Aprobado: 7 de junio 2021

Argentina Artavia Medrano Costarricense. Tiene una maestría en Educación en Derechos Humanos por CREFAL IIDH. Licenciada en Ciencias Políticas por la Universidad de Costa Rica. Es investigadora del Centro Agenda Joven en Derechos y Ciudadanía de la Universidad Estatal a Distancia y profesora e investigadora en la Escuela de Ciencias Políticas de la

Universidad de Costa Rica (Costa Rica). Trabaja los temas democracia, derechos políticos, participación política, juventud, construcción de ciudadanía, partidos políticos, derechos humanos. Corre electrónico: argentina.artavia@gmail.com

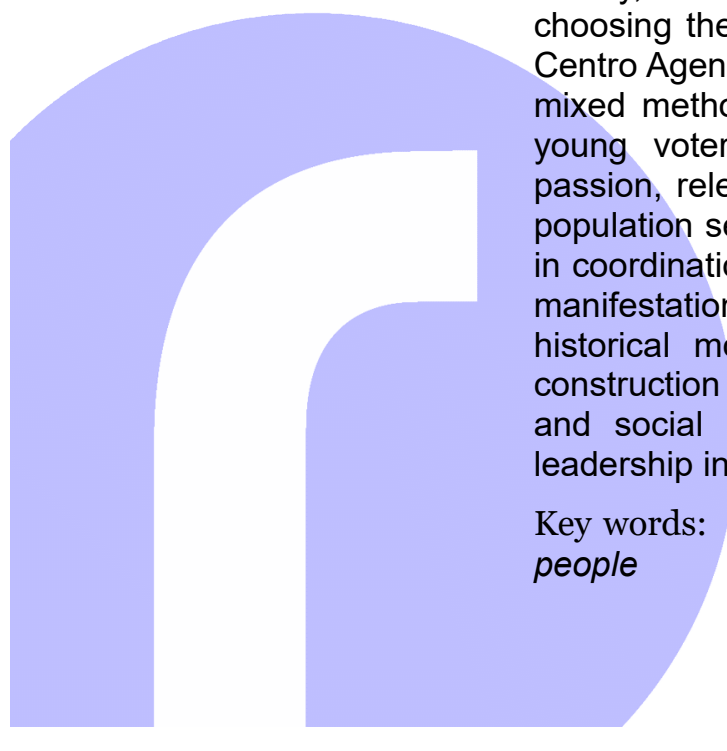
proceso. people
Resumen: Este artículo presenta los principales hallazgos de la experiencia de observación de un proceso sin precedentes en la historia político-electoral costarricense, por medio del cual la ciudadanía de Río Cuarto contó con la posibilidad de escoger el centro político y administrativo de su cantón y en el que el equipo del Centro Agenda Joven de la UNED participó como testigo de excepción. Mediante un enfoque de complementariedad metodológica, se determinó que la mayoría de las personas jóvenes electoras perciben la política con compromiso, entusiasmo e incluso pasión al relegar la indiferencia y el disgusto hacia actividades electorales. Este sector poblacional utiliza sus derechos políticos, tanto en aspectos logísticos, como de coordinación y fiscalización. Asimismo, la investigación registró diversas manifestaciones acerca de la responsabilidad que debían asumir las personas jóvenes en un momento histórico como este, en el que iniciaban el camino hacia la construcción de una identidad cantonal, quienes encontraron en los medios electrónicos y las redes sociales, herramientas de gran valía para asumir cuotas de responsabilidad y liderazgo en diversas actividades del

Palabras clave: plebiscito; democracia directa; derechos políticos; participación; personas jóvenes

\section{Plebiscite in Río Cuarto Costa Rican canton: an unprecedented exercise in direct democracy}

Abstract: This article shows the main findings obtained from the experience of observing an unprecedented process in the Costa Rican electoral political history, through which the citizens of Río Cuarto had the possibility of choosing the political and administrative center of their canton. The team of Centro Agenda Joven (UNED) participated as an exceptional witness. Using a mixed methods approach, it was possible to determine that the majority of young voters perceive politics with commitment, enthusiasm and even passion, relegating indifference and disgust towards electoral activities. This population sector uses its political rights, both in logistical aspects, as well as in coordination and supervision. Likewise, the investigation registered various manifestations about the responsibility that young people should assume in a historical moment like this, in which they began the path towards the construction of a cantonal identity, which they found in the electronic media and social networks, tools of great value to assume responsibility and leadership in the process.

Key words: plebiscite; direct democracy; political rights; participation; young 


\section{Introducción}

El 7 de abril de 2019, las personas ciudadanas que habitan los distritos de Río Cuarto, Santa Rita y Santa Isabel acudieron a las urnas por medio de un plebiscito, en votación directa y secreta, para decidir cuál de los tres sería la cabecera de cantón; el centro político y administrativo de las actividades locales. Esta consulta popular, convocada por el Tribunal Supremo de Elecciones (TSE), se convirtió en un acontecimiento histórico, no solo por ser la primera vez que organizaba y realizaba un evento de esa naturaleza, sino también por los altos niveles de participación de la ciudadanía.

1. Las personas que integraron el equipo de observación son: Nora González Chacón (politóloga y abogada. coordinadora del Centro), Mario Alberto Araya Pérez (antropólogo), Argentina Artavia Medrano (politóloga), Jorge Raúl García Fernández (sociólogo), Anthony García Marín (sociólogo), Roy González Sancho (psicólogo), Evita Henríquez Cáceres (abogada), Fernando Obando Reyes (antropólogo y comunicador social), Maikol Picado Cortés (psicólogo), Andrea Romero Brown (orientadora) y Bryan Vargas Vargas (arquitecto y sociólogo)
El presente artículo se enmarca en un proceso de investigación ${ }^{1}$ del Centro Agenda Joven de la Universidad Estatal a Distancia (UNED) de Costa Rica en la consulta popular ya mencionada; el equipo investigador participó como Observador Nacional con el cumplimiento de todos los deberes y las responsabilidades que estableció el TSE.

A partir de la pregunta generadora: ¿de qué manera participan las personas jóvenes en la consulta cantonal?, la investigación evidencia el quehacer del Observatorio Político Electoral Juvenil (OPEJ), se desarrolló un proceso permanente de observación sobre el comportamiento político electoral de las personas jóvenes y el ejercicio de sus derechos en los procesos consultivos y de elección popular a nivel nacional, cantonal y local.

El documento se ha organizado en tres componentes. En el primero de ellos, se describen algunos aspectos metodológicos, que guiaron la investigación; en el segundo, se presenta tanto una contextualización de la situación investigada como los diferentes hallazgos relacionados con ella. Finalmente, se presentan las conclusiones y las posibilidades para investigaciones futuras.

\section{Metodología}

En toda investigación, las decisiones metodológicas están estrechamente vinculadas con el objeto de estudio y las particularidades de la situación por investigar. En este caso específico, el comportamiento político electoral de las personas jóvenes y el ejercicio de sus derechos en los procesos consultivos y de elección popular abren oportunidades para visibilizar y analizar la manera en la que las personas jóvenes ejercen sus derechos políticos; además, permite caracterizar, auditar y analizar los espacios de participación y de toma de decisiones como ejercicio efectivo de su ciudadanía.

En consecuencia, se optó por desarrollar un estudio de tipo mixto de predominancia cualitativa, de tal manera que la complementariedad en la recolección de datos y su interpretación permitieran diferentes miradas de la situación investigada. La investigación cualitativa se realiza cuando existe un interés "por acceder a las experiencias, interacciones y documentos en su con- 
texto natural y en una manera que deje espacio para las particularidades" (Gibbs 2012, 13).

En cuanto al aporte cuantitativo, se elaboró un cuestionario a personas jóvenes votantes acerca de percepciones sobre los sentimientos que les generaba la política (compromiso, entusiasmo). Dichas acciones se realizaban durante los procesos electorales (logísticas, coordinación, fiscalización), interés por participar en el plebiscito, motivaciones para votar y opinión de la fiscalización del TSE en el proceso electoral. El equipo conoció e interpretó los datos con el fin de contar con una contextualización con base en lo que las personas jóvenes opinaban y percibían sin la necesidad de particularizar o individualizar la información.

Para administrar el cuestionario, se utilizó un muestreo no probabilístico con modalidad de cuotas. Se estableció al tomar en consideración cuatro variables de segmentación de la población joven de Río Cuarto. Se obtuvo mediante el Censo de Población y Vivienda de 2011 del INEC, a saber: rangos de edad, niveles de estudio, sexo y distrito de residencia. Con la combinación de estas cuatro variables, se decidió administrar el instrumento, en la modalidad de entrevista, a las 36 personas que reunían las características ya señaladas y que podían votar en la consulta popular.

Además, la predominancia de lo cualitativo se basó principalmente en la fenomenología con el propósito de visualizar las percepciones y los puntos de vista de las personas jóvenes participantes de la consulta- Todo ello se vinculó a las interacciones y la participación que han tenido en sus contextos inmediatos, así como el ejercicio de sus derechos. De acuerdo con Álvarez (2009), un estudio puede ser considerado fenomenológico si tiene especial interés en explorar el significado de las experiencias vividas cotidianamente por las personas.

Como toda investigación cualitativa, se cumplieron aspectos básicos como negociación de entrada al campo de trabajo. La UNED inscribió 11 personas, todas funcionarias e investigadoras del Programa Agenda Joven. Ese equipo interdisciplinario permitió realizar las actividades previstas en todos los centros de votación.

Luego de la inscripción formal, se realizaron varias sesiones de trabajo, en las cuales se brindó una descripción del proceso: qué es un plebiscito, integración de juntas receptoras, conformación del padrón electoral, ubicación de los centros de votación, conteo y escrutinio, características y datos importantes sobre el cantón, funciones de las personas observadoras y fiscales de partidos políticos acreditados, posiciones y valoraciones de los distintos actores participantes -dirigentes, comités y agrupaciones locales.

Los mismos investigadores son una parte importante del proceso de investigación, bien desde el punto de vista de su propia presencia personal como investigadores, bien 
desde el de sus experiencias en el campo y con la reflexividad que aportan al rol que desempeñan, pues son miembros del campo que es objeto de estudio (Gibbs 2012, 13).

La técnica de recolección fundamental consistió en la observación participante, la cual se rigió por los lineamientos y parámetros establecidos para todos los procesos desarrollados por el Programa Agenda Joven como instancia dedicada a la investigación académica.

En particular, para el desarrollo de esta técnica, el equipo de trabajo elaboró una guía de observación a fin de recolectar información acerca de: 1) caracterización de la participación y el comportamiento de las personas jóvenes en el proceso electoral vivido en los centros de votación, 2) protección y ejercicio de derechos políticos, y 3) confianza en la transparencia del proceso.

Como parte de la preparación del proceso de observación, se realizó una gira al cantón con el propósito de ubicar los centros de votación, las distancias entre ellos, así como las condiciones en los alrededores (caminos, infraestructura, entre otros) que permitieran contar con la información logística disponible y considerada necesaria para llevar a cabo la investigación.

Asimismo, el equipo contó con la oportunidad de entrevistar a algunas personas de la comunidad, quienes compartieron datos sobre el proceso previo a la obtención del cantonato y también sobre la organización que cada distrito había realizado de cara al plebiscito. En coordinación con el grupo de observación de la Escuela de Ciencias Políticas de la Universidad de Costa Rica, se desarrolló una serie de reuniones con los comités organizadores de cada distrito. En dichos encuentros, se contó con la oportunidad de escuchar a dirigentes, sus principales preocupaciones sobre las distintas necesidades del cantón, el por qué "merecían" ser la cabecera, sus principales propuestas y algunos detalles organizativos para el día de la votación.

La gira de reconocimiento permitió identificar personas clave en los distritos, la ubicación de cada integrante del equipo en cada centro de votación, así como la logística prevista por las personas organizadoras durante el proceso y en el momento de conocer los resultados.

Además, se realizó un seguimiento a las páginas electrónicas que los comités de cada distrito crearon con el propósito de estar al tanto de los mensajes y el ambiente que se iba generando previo al día de la votación.

Finalmente, se tomó la decisión seleccionar los sitios por distrito con mayor cantidad de votantes, debido a la ubicación de los centros de votación, cantidad de personas jóvenes en el padrón, la cantidad de integrantes del equipo de investigación y la imposibilidad de tener presencia en todos los centros de votación durante todo el día, principalmente por los tiempos de desplazamiento. 


\section{¿Por qué y para qué un plebiscito en Río Cuarto?}

El plebiscito es la consulta popular "mediante la cual los habitantes del cantón se pronuncian sobre un asunto de trascendencia regional, o se manifiestan sobre la revocatoria del mandato de un alcalde municipal" (TSE, 1998); fue establecido en el Código Municipal aprobado en 1998, como una de las facultades de los gobiernos locales. El Código señala que en cada una de estas consultas "deberán estar presentes los delegados que designe el Tribunal Supremo de Elecciones, quienes darán fe de que se cumplieron los requisitos formales exigidos [...] Los delegados del Tribunal supervisarán el desarrollo correcto de los procesos citados" (artículo 13).

Desde el año 1999, se realizó la primera consulta plebiscitaria ${ }^{2}$, la labor del TSE fue la de asesorar y fiscalizar los procesos convocados y organizados por los Concejos Municipales en distintos puntos del país al seguir los lineamientos establecidos en el Decreto N. ${ }^{\circ}$ 03-98 Manual para la Realización de 2. El plebiscito fue realizado para consultar a los distritos de Cóbano, Lepanto y Paquera si querían seguir perteneciendo a consultas populares a escala cantonal y distrital. El documento es una guía para que los Concejos Municipales pudieran elaborar los reglamentos para la realización de las consultas ciudadanas. De acuerdo con el numeral 2.2 del citado Manual, la consulta popular puede realizarse sobre cualquier asunto, siempre que cumpla con los siguientes requisitos: que el asunto por resolver sea de competencia Municipal, que no tenga un procedimiento debidamente reglado por la ley, que el resultado de la consulta pueda dar origen a un acto administrativo válido y eficaz de la autoridad Municipal y que la consulta verse sobre un asunto actual y de interés general para los habitantes de la comunidad (TSE, 1998, 2).

En el caso de Río Cuarto, la ley de creación del cantón, Ley N. ${ }^{\circ} 9440$ del 20 de mayo del 2017, no contempló la definición de la cabecera, por lo que debió impulsarse una reforma que dispuso que sería el TSE el encargado de organizar la consulta popular. Por primera vez en Costa Rica, el lugar donde se establecería la cabecera de un cantón se definiría mediante un plebiscito, dado que no había un concejo municipal que pudiera realizar la convocatoria.

En Costa Rica, las consultas locales son en principio organizadas por la municipalidad respectiva y la labor del Tribunal se limita a asesorarlas, fiscalizarlas y ante contención judicial, juzgarlas. Este caso es distinto por una sencilla razón: no existe una municipalidad que pueda hacerse cargo de ello. Por eso en esta oportunidad, el Tribunal se encargará también de la organización. De suerte tal que este plebiscito es ejemplo de dos cosas que normalmente se olvidan: primero, que el trabajo del Tribunal es permanente y segundo, que los procesos de decisión 
3. En la convocatoria al plebiscito, el Magistrado Luis Antonio Sobrado señaló "lo construimos a partir de un ordenamiento ya dado, pero para una cuestión muy especial, muy diferente, inédita en la historia nacional" ( https://www.facebook.com/TS ECostaRica/videos/234594347 491784) política local son la base misma de la democracia (TSE, 2019).

El plebiscito de Río Cuarto como proceso electoral presentó una serie de particularidades que es preciso señalar ${ }^{3}$.

- Debió definirse por Ley de la República la participación del TSE como el encargado de organizar, fiscalizar y escrutar la consulta ciudadana; es decir, el proceso electoral local como resultado de la inexistencia de un Concejo Municipal que lo realizara.

- Ante la falta de normativa específica, que regulara la consulta cantonal, el TSE decidió que aplicaría el Código Electoral, la Ley sobre Regulación del Referendo, el Manual sobre Consultas Populares, entre otras normativas, de manera que el proceso se desarrollara de acuerdo con los procedimientos que aplican en los otros procesos de elección que regulan, cuya efectividad y transparencia está garantizada. Las 16 juntas receptoras de votos estarían integradas en su totalidad por personas funcionarias regulares del TSE, en tanto que representantes de los partidos políticos fiscalizarían el proceso.

En el proceso de investigación de este cantón y con las particularidades anteriormente señaladas, el interés se centró en conocer la participación e inclusión de las personas jóvenes en el proceso, así como la credibilidad en las instituciones político electorales en el contexto político electoral del plebiscito.

De acuerdo con González $(2017,98)$

La credibilidad política, propiamente dicha, no sería una calidad que una instancia adquiere solamente por seguir dicho dominio de lo social, sino que se aproximaría más a un resultado de procesos que han manifestado ser meritorios de confianza ante la opinión pública o especializada. Siguiendo los planteamientos de Zamitiz (1998), la credibilidad política en un sistema o instancia política se adquiere cuando se han logrado conseguir y mantener en el tiempo opiniones o valoraciones a favor de sí, que le acreditan como confiable.

El grupo de observación político electoral del Centro Agenda Joven documentó, sistematizó y analizó la información obtenida para mostrar una detallada caracterización de la participación de las personas jóvenes, así como el ejercicio de sus derechos políticos mediante la utilización de una de las estrategias de la democracia directa, por considerar que la participación y la inclu- 
sión son aspectos de gran relevancia en el análisis del comportamiento político de las personas (González, 2017).

\section{Las personas jóvenes y el plebiscito}

De acuerdo con la Ley de la Persona Joven, "se reconoce a la persona joven como actor social, cultural, político y económico, de importancia estratégica para el desarrollo nacional". Los datos del TSE sobre este sector poblacional (que va desde los 18 hasta los 35 años) habilitado para participar en el proceso de consulta en Río Cuarto por distrito electoral, se presentan en la tabla 1.

Tabla 1. Río Cuarto: Cantidad de votantes entre los 18 y 35 años, según distrito electoral (abril 2019)

\begin{tabular}{lcc}
\hline \multirow{2}{*}{ Distrito electoral } & \multicolumn{2}{c}{ Categorías de edad } \\
\cline { 2 - 3 } & De $\mathbf{1 8}$ a $\mathbf{2 5}$ años & De 26 a 35 años \\
\hline Colonia Agrícola del Toro & 31 & 52 \\
La Españolita & 48 & 56 \\
La Tabla & 95 & 124 \\
La Victoria & 88 & 91 \\
Los Ángeles & 21 & 21 \\
Pata de Gallo & 2 & 8 \\
Río Cuarto & 412 & 460 \\
San Rafael de Río Cuarto (Cucaracho) & 83 & 95 \\
San Vicente & 17 & 18 \\
Santa Isabel & 203 & 185 \\
Santa Rita & 360 & 333 \\
\hline Total & $\mathbf{1 3 6 0}$ & $\mathbf{1 4 4 3}$ \\
\hline
\end{tabular}

Fuente: Estadísticas del Tribunal Supremo de Elecciones.

De acuerdo con los datos de la tabla 1, de las 2803 personas jóvenes que habitan el cantón de Río Cuarto, el 48,52 \% tienen entre 18 y 25 años; el $51,48 \%$ tienen una edad entre 26 y 35 años. Además, esta información también permitió ubicar a los centros de votación con el mayor número de personas jóvenes votantes, lo cual se utilizó para distribuir al equipo de investigación.

Según el testimonio oral de algunas personas que habitan el cantón, el interés y la motivación por un proceso democrático sin precedentes fueron las principales causas de la reducción de los porcentajes de abstencionismo registrados en los últimos procesos electorales, particularmente entre la población joven ${ }^{4}$.

4. Según resolución $\mathrm{N} .^{\circ} 2688$ E11-2019 del 23 de abril de 2019, el TSE señaló que el porcentaje total de participación electoral del Según se indicó en la sección de metodología de este artículo, se efectuaron consultas a las personas jóvenes sobre aspectos considerados básicos acerca de su rol en la política. Para ello, se empleó un cuestionario administrado plebiscito alcanzó el $72,38 \%$. 
por entrevista. Seguidamente, se detallan los resultados organizados de esa indagación.

Como parte del cuestionario, se utilizó una escala Likert de intensidad acerca de las emociones que suscitaba la política en el sector poblacional entrevistado. En la tabla 2 se muestran los porcentajes correspondientes a las emociones destacadas por el equipo de investigación y la distribución de las respuestas según la escala Likert empleada.

Tabla 2. Percepción, en porcentajes, de las personas jóvenes votantes sobre las emociones que le provoca la política

\begin{tabular}{lrrrrrr}
\hline Categorías & Nada & Poco & Algo & Bastante & Mucho & Total \\
\hline Entusiasmo & 5,6 & 22,2 & 27,8 & 22,2 & 22,2 & $\mathbf{1 0 0 , 0}$ \\
Indiferencia & 58,3 & 19,4 & 11,1 & 5,6 & 5,6 & $\mathbf{1 0 0 , 0}$ \\
Cansancio & 55,6 & 8,3 & 19,4 & 8,3 & 8,3 & $\mathbf{1 0 0 , 0}$ \\
Desconfianza & 27,8 & 13,9 & 33,3 & 8,3 & 16,7 & $\mathbf{1 0 0 , 0}$ \\
Compromiso & 8,3 & 13,9 & 5,6 & 30,6 & 41,7 & $\mathbf{1 0 0 , 0}$ \\
Pasión & 19,4 & 11,1 & 22,2 & 22,2 & 25,0 & $\mathbf{1 0 0 , 0}$ \\
Disgusto & 52,8 & 11,1 & 25,0 & 8,3 & 2,8 & $\mathbf{1 0 0 , 0}$ \\
\hline
\end{tabular}

Elaboración propia con base en el cuestionario aplicado en el Plebiscito Río Cuarto - abril 2019.

5. Mediante resolución $\mathrm{N}$. 2688-E11-2019 del 23 de abril de 2019, el TSE oficializó los resultados generados durante el plebiscito. El distrito de Río Cuarto obtuvo 2235 votos, seguido de Santa Rita con 2117 y Santa Isabel con 87 votos; se contabilizaron 12 votos nulos y 2 en blanco. El porcentaje total de participación electoral alcanzó el $72,38 \%$.
En la tabla 2 se nota que más de un $70 \%$ manifestó que les generaba mucho o bastante compromiso y más de un $40 \%$, bastante o mucho entusiasmo. Dichos datos respaldan aquellos que posteriormente fueron dados a conocer sobre la participación de las personas jóvenes en la consulta. Asimismo, conviene resaltar que las respuestas vinculadas a las emociones consideradas como negativas (indiferencia, cansancio y disgusto) no representan nada para más de la mitad de las personas. Cabe destacar que el 33,3\% señaló que la política le causa algo de desconfianza, frente a un $41,7 \%$ que indica poco o nada en esa categoría.

También, se les consultó sobre las acciones que realizaban durante los procesos electorales y, en su mayoría, consideraron que las personas jóvenes apoyaban actividades logísticas y de coordinación; más del $60 \%$ opinó que realizan poca o ninguna labor de fiscalización, tal y como se muestra en la tabla 3.

Conviene resaltar que en la categoría "van a votar" se alcanzó un 69,4 \% entre las categorías (algo, bastante y mucho). Este dato resultó muy cercano a la participación total reportada por el TSE para el plebiscito ${ }^{5}$. 
Tabla 3. Percepción, en porcentajes, de las actividades en las que participan las personas jóvenes

\begin{tabular}{|c|c|c|c|c|c|c|c|}
\hline $\begin{array}{l}\text { Las personas } \\
\text { jóvenes }\end{array}$ & Nada & Poco & Algo & Bastante & Mucho & NS & Total \\
\hline Van a votar & 2,8 & 25,0 & 25,0 & 25,0 & 19,4 & 2,8 & 100,0 \\
\hline $\begin{array}{l}\text { Entregan } \\
\text { documentos e } \\
\text { información }\end{array}$ & 5,6 & 25,0 & 25,0 & 27,8 & 16,7 & 0,0 & 100,0 \\
\hline $\begin{array}{l}\text { Fiscalizan } \\
\text { actividades } \\
\text { electorales }\end{array}$ & 33,3 & 33,3 & 19,4 & 11,1 & 2,8 & 0,0 & 100,0 \\
\hline $\begin{array}{l}\text { Apoyan } \\
\text { actividades } \\
\text { logísticas } \\
\text { Coordinan }\end{array}$ & 2,8 & 16,7 & 33,3 & 22,2 & 25,0 & 0,0 & 100,0 \\
\hline $\begin{array}{l}\text { actividades } \\
\text { electorales }\end{array}$ & 8,3 & 30,6 & 27,8 & 19,4 & 13,9 & 0,0 & 100,0 \\
\hline
\end{tabular}

En cuanto a la opinión de las personas entrevistadas acerca de su interés por participar en el proceso, las respuestas fueron mayoritariamente positivas. Para esta pregunta, el equipo investigador consideró relevante diferenciar las respuestas por sexo, según se muestra en la tabla 4 .

Tabla 4. Percepción, en porcentajes, del interés de las personas jóvenes de participar en el plebiscito, según sexo

\begin{tabular}{|c|c|c|c|}
\hline \multirow{2}{*}{$\begin{array}{l}\text { Interés de participar } \\
\text { en el plebiscito }\end{array}$} & \multicolumn{2}{|c|}{ Sexo } & \multirow{2}{*}{ Total } \\
\hline & Hombre & Mujer & \\
\hline Nada & 16,7 & 5,6 & 11,1 \\
\hline Poco & 11,1 & 0,0 & 5,6 \\
\hline Algo & 16,7 & 16,7 & 16,7 \\
\hline Bastante & 38,9 & 22,2 & 30,6 \\
\hline Mucho & 11,1 & 55,6 & 33,3 \\
\hline NS & 5,6 & 0,0 & 2,8 \\
\hline Total & 100,0 & 100,0 & 100,0 \\
\hline
\end{tabular}

En general, un 63,9 \% señaló que consideraba que las personas jóvenes tenían bastante o mucho interés de participar. Según los datos de la tabla 4 , la 
opinión de las mujeres consultadas es mucho más proclive a la participación $(77,8 \%)$ que la de los hombres $(50,0 \%)$.

Con el propósito de ahondar en el porqué del interés en la participación, el $45,9 \%$ de las personas indicó que su motivo para votar era "mejorar el cantón", mientras que el 47,6\% manifestó que era tanto por el derecho como por el deber de votar. El 6,5\% restante indicó que votaría por los compromisos o promesas de campaña u otros motivos no especificados.

En consecuencia, se mantiene la concepción del voto -como un derecho y un deber- y como elemento fundamental de la cultura cívica y política con la intención de incidir en la comunidad para mejorarla.

Otra de las preguntas realizadas fue si consideraban que el TSE era eficiente en la fiscalización del proceso electoral. En la tabla 5 se presentan los resultados de la percepción por parte del sector poblacional consultado.

Tabla 5. Percepción sobre la fiscalización que realiza el Tribunal Supremo de Elecciones en el Plebiscito Río Cuarto (abril 2019)

\begin{tabular}{lccc}
\hline \multirow{2}{*}{ Categoría } & \multicolumn{2}{c}{ Sexo } & Total \\
\cline { 2 - 3 } & Hombre & Mujer & \\
\hline Nada & 11,1 & 0,0 & $\mathbf{5 , 6}$ \\
Poco & 5,6 & 5,0 & $\mathbf{5 , 6}$ \\
Algo & 16,7 & 0,0 & $\mathbf{8 , 3}$ \\
Bastante & 11,1 & 0,0 & $\mathbf{5 , 6}$ \\
Mucho & 55,6 & 88,9 & $\mathbf{7 2 , 2}$ \\
NS & 0,0 & 5,6 & $\mathbf{2 , 8}$ \\
\hline Total & $\mathbf{1 0 0 , 0}$ & $\mathbf{1 0 0 , 0}$ & $\mathbf{1 0 0 , 0}$ \\
\hline
\end{tabular}

Elaboración propia con base en el cuestionario aplicado.

De nueva cuenta, el equipo investigador decidió diferenciar la percepción por sexo en cuanto a la labor del TSE se refiere. El 77,8 \% de las personas resaltó la labor del órgano electoral durante el proceso al calificar la eficiencia en las categorías "bastante" y "mucho". En este aspecto, existen diferencias en cuanto al sexo, porque el $88,9 \%$ de las mujeres escogió la categoría "mucho", mientras que solo el 55,6 \% de los hombres coincidió con esa percepción.

\section{Una mirada fenomenológica del contexto para el plebiscito}

Como ya es costumbre en Costa Rica, las juntas receptoras de votos se instalan en centros educativos. En el cantón de Río Cuarto, el TSE decidió utilizar los mismos centros y juntas que en las elecciones presidenciales de 2018 para facilitar el proceso a las personas electoras y fue en esos lugares en los 
que se ubicó el equipo investigador; se registró que los centros contaban con una infraestructura en buenas condiciones.

Cada junta se ubicó en aulas amplias, con buena ventilación e iluminación, la pintura no estaba dañada, algunas canoas estaban un poco oxidadas y algo desprendidas; pero, el daño no era tan notable; los pisos y los cielorrasos no se veían deteriorados. En otros centros, las aulas eran espacios pequeños y en apariencia viejos; sin embargo, resultaban agradables a la vista. Casi todos los centros educativos contaban con una plaza en las cercanías, que era utilizada para diversos fines; algunos para actividades deportivas o, como en ese día, se destinó a colocar toldos y mesas para recibir a las personas votantes.

El acceso a las instituciones educativas era fácil, amplio, con rampas para personas con discapacidad, sin gradas desde la entrada hasta los baños; no obstante, algunas aulas tenían puertas muy estrechas que impedían la entrada de una silla de ruedas, tal como pudo observarse en una de las aulas de la escuela de Río Cuarto. Una de las observadoras en ese centro pudo atestiguar que, luego de muchos esfuerzos, las personas integrantes de la mesa receptora de votos acondicionaron un espacio afuera del aula para que la persona pudiera emitir su voto.

En las zonas aledañas, la mayor parte de las estructuras correspondían a estructuras de la iglesia o templos, pequeños comercios (abastecedores, sodas). Las zonas verdes bien podadas y arregladas, en algunas comunidades, incluso, contaban con algunas bancas con mesas en cemento. Resultó evidente observar el predominio de espacios abiertos sin mallas perimetrales, ni algún otro tipo de barrera que dificultara su acceso.

Las escuelas ubicadas en el centro de Río Cuarto o Santa Rita contaban con todas las comodidades, infraestructura y acceso sin inconvenientes; ofrecían todo tipo de comercio, tales como pequeños hoteles, abastecedores, sodas, ferreterías, cafeterías, restaurantes, carnicerías, etc. En el caso de la escuela de Santa Rita, había una sucursal bancaria, un centro comunal y una plaza de considerables dimensiones; en las cercanías de la escuela de Río Cuarto, se ubicaba la Cruz Roja, una estación de bomberos, un parque, la parroquia y el centro comunal. Por el contrario, cerca de la Escuela La Victoria (en el distrito de Río Cuarto), solo se encontraban una iglesia, un pequeño abastecedor y una plaza para partidos de béisbol, que principalmente la utilizaba la población migrante nicaragüense que trabaja en las plantaciones de piña.

La Escuela La Españolita en Santa Isabel está ubicada en un lugar de paso entre el corredor noratlántico y el centro del distrito sobre la carretera Chilamate - Vuelta Kooper. El uso del suelo está dedicado a la ganadería y la plantación extensiva de piña con algunos pequeños comercios y viviendas aisladas. La escuela está ubicada a unos 150 metros de la carretera; un camino de lastre los conecta hasta el punto donde está la institución, la iglesia, una cancha de fútbol y algunas viviendas. El sector tiene dificultades de transporte público. Además, existen lugares muy alejados de los cuales es 
difícil salir; estas condiciones pudieron incidir en la posibilidad de ejercer el derecho al voto.

Las diferencias entre los distritos son muy evidentes en todo el territorio no solo en función de las condiciones materiales y de infraestructura, sino también en lo que implican para el desarrollo económico y el bienestar de vida de sus habitantes. Las amplias zonas dedicadas al cultivo de piña y a la ganadería, los sitios de difícil acceso, la infraestructura desigual (caminos de lastre en algunas comunidades y carreteras asfaltadas en otras) impiden el desplazamiento rápido y seguro entre poblados y los servicios básicos centralizados en las principales comunidades reflejan las pocas oportunidades de muchas de las personas jóvenes. Todo ello se convierten en un reto importante para el nuevo cantón alajuelense.

La actividad económica define en mucho las condiciones de la participación de las personas y las posibilidades de desarrollo. Por ejemplo, en las comunidades donde el cultivo de la piña es la única posibilidad de empleo, el acceso a servicios se limita y las condiciones de infraestructura y caminos son difíciles, porque no constituyen elementos que motiven la participación y menos de las personas jóvenes, quienes no encuentran oportunidades realmente atractivas.

Es notable destacar que las personas de la comunidad se conocen, se saludan y aprovechan para conversar un rato, tanto al ingreso del centro educativo como cuando han terminado de votar. Así, se observó un ambiente de mucha cordialidad. Las personas jóvenes, que llegaron al centro de votación, lo hacían de forma rápida y al terminar se retiraban; algunos se quedaban a saludar a sus amistades y luego se iban.

En lo que se refiere a signos externos en el lugar de votación (afiches, volantes, pancartas, entre otros) fue notoria la diferencia en cuanto a la cantidad y calidad entre los distritos. Las agrupaciones contaban con toldos y camisetas que usaban las personas que prestaban colaboración; además, los carros portaban calcomanías y banderas. En Santa Rita y Río Cuarto, el material provenía de imprentas y serigrafías, mientras que las banderas de Santa Isabel eran más artesanales, puesto que se elaboraron con manta y tinta. En este último lugar, la diferencia de recursos y propaganda se evidenció en un comentario que presenció una de las personas integrantes del equipo observador, cuando un grupo de menores de edad comenzó a jugar con globos y a estallarlos. En ese momento, una señora del lugar les dijo: "no me revienten las bombas; las compré con mi plata y salen muy caras, por favor, no las revienten" (Diario de campo, Santa Isabel, 2019).

Otro aspecto destacado por el equipo observador consiste en que solo Santa Rita entregó material impreso (volantes), así como el hecho de que prácticamente no se encontraron evidencias de signos externos en otros sitios cercanos al centro de votación, donde se ubicaron los toldos que se convirtieron en centros de operaciones y transporte de votantes. 
No se identificó algún tipo de grafiti, mural o símbolos relacionados con participación política o similar. En las escuelas existían dibujos de paisajes rurales o algún material alusivo a alguna celebración especial, pero ninguno mantenía relación explícita con el evento electoral. Únicamente, en la escuela de San Rafael, había un letrero con la bienvenida a las personas que acudieron a votar.

\section{Un hito en la historia político-electoral costarricense}

El día del plebiscito, la jornada inició conforme lo previsto y en algunos centros de votación fue notoria la presencia de personas con intención de votar, aún antes de que las urnas abrieran. Las primeras personas en llegar a los centros de votación fueron principalmente hombres, algunos de los cuales venían en motocicletas. Casi en todos los centros de votación, la participación fue significativa entre las 8:20 a.m. y las 10:00 a.m. en las que hubo una mayor afluencia, así como de 2:15 p.m. a 3:30 de la tarde. Las personas acudían al centro de votación por sus propios medios de transporte tales como motos y automóviles; algunas también utilizaron los medios que dispusieron personas de la comunidad o de los equipos de campaña para organizar el traslado al centro de votación.

En la Escuela La Victoria en Santa Rita, las personas jóvenes, que asistieron a votar, fueron hombres, mayores de 25 años; en su mayoría, llegaron en motocicleta, mientras que las mujeres jóvenes acudían en el carro con su pareja o en los carros dispuestos por habitantes de la comunidad para el traslado de las personas de sus casas al centro de votación.

También, se observó la participación de personas menores de 18 años, quienes entregaban la documentación y servían de "guías de votación", principalmente mujeres. En casi todos los centros de votación, contaban con la función de identificar, en un padrón impreso, cuáles personas habían ejercido su voto. De esa forma, organizaban los viajes en los cuales iban a buscar a aquellas personas que aún no lo habían hecho.

En la escuela de Río Cuarto, uno de los puntos fuertes fue la organización y la movilización de votantes. Contaban con tres mecanismos de control de padrón: en una primera lista, tachaban el nombre con marcadores de diferentes colores; otro grupo de jóvenes tenía computadoras portátiles y celulares con una aplicación en la cual registraban quiénes habían votado. Los jóvenes guías debían anotar en unas boletas el número de mesa y de votante y, de inmediato, reportarlo a quienes resguardaban el padrón para actualizar la base de datos. Ante la pregunta de las observadoras a uno de los dirigentes, reconoció que eso se debía a una organización muy bien detallada y que contaban con un padrón, una base de datos que actualizaban constantemente y un centro de control de datos en donde registraban el control de todo el proceso.

Las personas encargadas de incentivar el voto por el Distrito de Santa Rita se acercaban e indicaban que "los servicios públicos estarían más cerca". 
Además, las personas guías de Río Cuarto enfatizaban en que fue esa comunidad la que lideró la lucha por el cantonato y por eso "merecen ser la cabecera"; sin embargo, a pesar de la vehemencia con que se intentaba disputar cada voto, no se registraron incidentes de conductas agresivas entre los grupos adversarios, sino que prevaleció un ambiente festivo.

Usualmente, los grupos de jóvenes con edades entre los 15 y 25 años estaban identificados con camisetas de Santa Rita o de Río Cuarto y se encargaban de la actividad logística: guiar a las personas a la junta receptora o acompañarlas de sus casas al centro de votación con el apoyo de la revisión del padrón descrita anteriormente.

De manera similar, en Santa Isabel, aunque evidentemente con menos recursos, una de las personas se encargó de revisar el padrón para identificar quiénes aún no habían votado a fin de ubicar su lugar de residencia y coordinar su traslado a la junta receptora de votos. Igualmente, se identificó a una menor de edad colaboradora en el proceso, quien repasaba una lista de personas pendientes de llamar y, junto con otra, se ponían de acuerdo para pedir direcciones de personas conocidas y traerlas al centro de votación.

En general, las personas jóvenes, que llegaban a ejercer el voto, sobre todo hombres, lo hacían en su ropa de trabajo, en botas, con la ropa sucia; algunos se disculpaban con las personas integrantes de las juntas receptoras por su presentación personal e inmediatamente mencionaban que venían de trabajar, como suele suceder en estos contextos, en que muchas personas trabajan los domingos en diferentes actividades agropecuarias y portan ropa acorde con la actividad que realizan.

A medida que avanzaba el tiempo y se acercaba la hora de cierre, la urgencia por atraer votos aumentó. Se volvió constante escuchar gritos que decían que había carros dispuestos a ir a buscar a la gente ausente a los centros electorales; hacían un control cruzado y verificaban cuál integrante de la familia no había votado con la intención de facilitar su traslado al centro de votación.

Es importante mencionar que todas las personas en los centros educativos, principalmente personas jóvenes, acompañaron y recibieron con mucho cariño y respeto a las personas adultas mayores, que asistieron a votar; les brindaron asistencia a la hora de ubicar la junta receptora y emitir el voto. En la escuela de Río Cuarto, se registró el siguiente testimonio oral: "era una forma de agradecerles y reconocerles como los gestores del movimiento por el cantonato. Si no fuera por ellos, no estaríamos viviendo esto". (Diario de campo, Río Cuarto, 2019).

\section{El ejercicio de los derechos políticos de las personas jóvenes}

A lo largo del día, fue poco usual ver a personas jóvenes que utilizaran los espacios recreativos, a pesar de que la mayoría de los centros de votación 
tenía al frente una plaza de deportes. Quienes estaban en el centro o sus alrededores eran personas de la comunidad que llegaban a votar o estaban colaborando en las labores propias del proceso electoral; en su mayoría, se trataba de personas menores de edad, principalmente mujeres.

Durante el proceso, las personas jóvenes asumieron una labor muy visible y no solo en lo logístico. Algunas de ellas manifestaron ser una generación con un importante liderazgo en la comunidad. Incluso, plantearon que "este era su momento", tenían claridad de lo que querían hacer y hacia dónde querían llevar al cantón, cuál era su responsabilidad en lo que llamaron un "momento histórico".

Un aspecto considerado como fundamental fue el manejo y el acceso a medios electrónicos y redes sociales, lo que les permitió no solo participar en la organización, sino también asumir liderazgo en algunas actividades (control del padrón, comunicación, por ejemplo). Las personas jóvenes se relacionaron entre sí para ejercer un liderazgo con el apoyo de la tecnología.

En ninguno de los centros de votación se observó algún aspecto que alertara sobre limitaciones para el ejercicio de los derechos políticos de las personas jóvenes. Puede decirse que existieron todas las condiciones necesarias para que se ejerciera el voto; las instalaciones e infraestructura eran, en su mayoría, las adecuadas.

En un testimonio oral, un joven dirigente del distrito de Río Cuarto fue enfático al señalar que "aquí no va a encontrar a nadie que diga que el Tribunal no sabe hacer las cosas; todos estamos seguros de que lo están haciendo bien". En otra conversación, suscitada en el mismo lugar, se dijo que "el TSE tenía todo muy controlado y que ellos solo debían ir a sacar la gente para que votaran".

Adicionalmente, debe señalarse que las personas jóvenes, quienes formaban parte de los grupos de observación del proceso, tanto estudiantes de la UCR, como de la UNED, a lo largo del día, disfrutaron de la libertad de observar y fotografiar todos los espacios permitidos. Las personas delegadas del TSE casi en todos los sitios brindaron facilidad en cuanto al acceso a la información o la permanencia en los espacios. En los alrededores de los centros de votación, las personas jóvenes colaboradoras de los movimientos se desplazaron y realizaron sus actividades a lo largo del día sin inconveniente alguno; quienes llegaron a ejercer el voto no hallaron inconveniente o contratiempo. Tampoco se observó algún incidente con las personas que estaban en la junta receptora de votos ni con las personas que fiscalizaban el proceso.

Se observó que gran cantidad de mujeres jóvenes llegaba con sus hijos e hijas o acompañadas de personas menores de edad a ejercer el voto. Fue notoria la presencia de mujeres jóvenes con sus madres o sus parejas, quienes se mantuvieron a la par de ellas en todo momento.

Por su parte, las personas jóvenes movilizaron más a sus pares, lo que da cuenta de la capacidad de establecer redes y atraerlas a las actividades que 
6. En Costa Rica, el "Día E" es el nombre con el que los grupos de estrategia de las campañas políticas identifican el día de las elecciones. les interesan. "Vine porque usted me lo pidió”, “¿Vio? No le quedé mal”, "ya le cumpli”. Esas fueron algunas de las frases que se escucharon por parte de jóvenes una vez que salían del recinto de votación (Diario de campo, Río Cuarto, 2019).

\section{El inicio del camino hacia la identidad cantonal}

El plebiscito y todo el proceso de campaña desarrollado, según lo indicaron algunos dirigentes comunales, son el inicio para la construcción de la identidad cantonal, porque les deja la capacidad instalada de ser "electores" y la posibilidad de tomar sus propias decisiones. Anteriormente, se percibían como un distrito olvidado de Grecia, o la comunidad que por conveniencia se acercaba a San Carlos; ahora, tenían la posibilidad de asumirse como una comunidad con oportunidades y desafíos que debían resolver entre las personas habitantes y consideraban que eso los podía unir más.

El día anterior a la consulta, en Río Cuarto, dos líderes del movimiento fueron los que recibieron al equipo observador, ambos hombres. El primer dirigente, un hombre adulto, realizó una referencia a la historia de Río Cuarto. Señaló que cada cuatro años los llamaban de Grecia para organizar la municipalidad, pero que ahora peleaban por su realidad en un contexto político diferente. Mencionó que un descubrimiento en el proceso de plebiscito consistió en que la política es alianza, tomar decisiones, luchar y ponerse de acuerdo. No veían la política como "eso feo político partidario", sino que se tomó en cuenta a personas de diferentes partidos y de diferentes comunidades del cantón. También, señaló que, al inicio del proceso, estaban muy enojados, porque se dijo desde el principio que Río Cuarto era la cabecera y que el proceso convocado por el TSE no les había gustado; sin embargo, "ahora todo el pueblo de Río Cuarto se metió en el asunto". Se refirió a la organización para el Día $E^{6}$. Indicó que había personas encargadas de los guías, alimentación y transportes, en su mayoría adolescentes y mujeres jóvenes (Diario de campo, Río Cuarto, 2019).

El segundo dirigente, un hombre joven, se presentó como el representante del cantón y jefe de campaña electo por el equipo de trabajo. Señaló que trabajaron muy duro desde el inicio del proceso para lograr el cantonato y que mucho de lo que se hizo fue muy artesanal (como ejemplo mencionó el hecho de que cada una de las 57 cartas enviadas a las personas diputadas fueron elaboradas en hojas recortadas de un cuaderno y redactadas a mano; según el joven, eso dice mucho de lo que la gente de Río Cuarto está dispuesta a hacer por su comunidad). Sobre el proceso, mencionó que comenzaron la campaña con un año de anticipación al plebiscito y que, producto de ello, se había logrado construir una identidad propia. "Nada está por encima de la causa Río Cuarto cabecera". Aseguró que la gente del distrito veía esta campaña como la más importante de su vida; incluso, dijo que había "mucho jubilado que en su vida ha votado y en esta ocasión de seguro sí vota". Expresó que su mayor ilusión era poder llevar a su abuelo a votar, puesto que era uno de los pioneros en la lucha por el cantonato y había estado muy en- 
fermo; creía que participar activamente era la mejor forma de agradecerle y reconocerle su trabajo y todo lo que había hecho por la comunidad. "Lo mejor será poder decirle que ganamos la cabecera. La campaña ha sido muy emotiva. Todo es por Río Cuarto" (Diario de campo, Río Cuarto, 2019).

Como es evidente, todo proceso comunitario tiene detractores. No todas las personas asimilaron de buena gana la manera en la que el proceso se iba a realizar. Como en la ley no se definió la cabecera y por Constitución todo proceso electoral debe ser organizado y regulado por el TSE, lo que debió asumirse como cualquier otro proceso, algunos de los habitantes lo vieron como "la gente de afuera que viene a decirles qué hacer y cómo" (Diario de campo, San Rafael, 2019). Por ejemplo, un hombre adulto mayor le indicó a uno de los observadores que le parecía que el plebiscito "había sido un error", porque la ley de creación del cantón debió establecer la cabecera ${ }^{7}$. Mencionó las rencillas o conflictos históricos entre los pobladores de Río Cuarto y Santa Rita, los cuales "se han extendido y profundizado debido al proceso" (Diario de campo, San Rafael, 2019).

En reuniones con los comités organizadores de cada distrito se tuvo la oportunidad de escuchar a los dirigentes y sus principales preocupaciones sobre las distintas necesidades del cantón; el por qué "merecían" ser la cabecera, la exposición de las principales propuestas, así como los principales detalles organizativos para el día de la elección. Desde ese momento, empezaron a visibilizarse las diferencias de los espacios ocupados por las personas jóvenes.

En Santa Rita, las personas fueron convocadas al salón comunal; sin embargo se destacó la presencia de una gran cantidad de personas jóvenes adolescentes y mujeres jóvenes, quienes dirigieron la reunión y dieron los principales discursos fueron hombres adultos. Un líder de la comunidad ofreció el discurso de fondo con el que justificó las razones por las cuales debían votar por Santa Rita como cabecera de cantón, entre ellas que era un distrito equidistante a todo el cantón; tenían experiencia en organización por cuanto varios miembros de la comunidad integraban el Consejo de Distrito y además eran el distrito con mayor densidad de población. También, mencionaron que había mejores opciones de desarrollo por su cercanía con la nueva carretera Chilamate - Vuelta Kooper y con el puerto de Moín, lo cual era una ventaja puesto que podría utilizarse en la creación de zonas francas desconcentradas y generar, de esta forma, trabajo y desarrollo. Asimismo, indicaron que se requería generar fuentes de empleo y que, para ello, habían hecho una alianza con la Universidad Técnica Nacional para que formaran técnicos en manejo de aguas y desechos industriales. En otro orden, plantearon la necesidad de cambiar el tipo de cultivo hasta ahora realizado, porque "es lo único que nos puede ayudar a dejar de ser agricultores toda la vida", en clara referencia al cultivo principal de la zona: la piña (Diario de campo, Santa Rita, 2019).

\author{
7. En la convocatoria al \\ plebiscito, el Magistrado \\ Sobrado manifestó: "No creo \\ que sea un error que al \\ Tribunal le corresponda \\ organizar esta consulta, porque \\ no hay ninguna municipalidad \\ que corresponda al nuevo \\ cantón de Río Cuarto. La \\ Asamblea Legislativa bien \\ pudo haber dispuesto la \\ cabecera del cantón en el acto \\ legislativo, no lo hizo y decidió \\ procesar la diferencia de \\ criterio que puede haber en la \\ comunidad de la mejor manera \\ posible, que es una consulta a \\ los propios vecinos, a los \\ propios interesados. De aquí \\ emerge una decisión \\ democrática, respaldada por la \\ mayoría de las personas" \\ ( https://www.facebook.com/TS \\ ECostaRica/videos/234594347 \\ 491784)
}


8. Los partidos políticos acreditaban fiscales generales y fiscales en cada junta receptora, si así lo consideraban conveniente. De esta manera, Acción Ciudadana acreditó 29 fiscales; Liberación Nacional, 16; Republicano Social Cristiano, 46 y Unidad Social Cristiana, 20 para un total de 111 fiscales acreditados. Fuente: TSE.

9. Para el Director General del Registro Electoral del TSE, Héctor Fernández Masís, "esta jornada de votación, este plebiscito es inédito para nosotros también como organización electoral; es una de las pocas oportunidades que tenemos de estar "de ese otro lado del mostrador", de poder ser miembros de mesa activos, abrir la mesa y cerrar" (Recuento del proceso https://www.facebook.com/TSE CostaRica/videos/5508223587 77938)

\section{Un recuento político}

La ausencia de información oportuna, que aclarara dudas y explicara adecuadamente los detalles del proceso, parece que es una debilidad de la organización comunal, puesto que el TSE aseguró haber realizado varios talleres desde que se anunció la fecha del plebiscito. Aquí, se manifiestan las distintas percepciones de los dirigentes de algunas comunidades en contraposición con las del resto.

Aun cuando los habitantes de distintas comunidades quisieron restarle importancia a la presencia de los partidos políticos nacionales, fue muy evidente no solo en la acreditación de los fiscales ${ }^{8}$, sino también en la visita de personas diputadas a diferentes recintos, tal como el equipo investigador lo constató.

Por sí misma, la presencia de representantes de partidos políticos no implicó un riesgo ni una amenaza a la participación o autonomía de los grupos comunitarios organizados; sin embargo, es preciso no tratar de invisibilizar su injerencia, principalmente, de cara a futuros procesos de elecciones municipales en los que se escogerán las autoridades del nuevo cantón.

A pesar de la importancia del proceso, fue evidente que no despertó el interés del resto del país. Únicamente, algunos medios como NC11, Radio Columbia y Radio Nacional, así como el Canal 14 o Radio Santa Clara (dos medios locales), acudieron a darle cobertura al proceso.

En cuanto a la labor del TSE, a pesar de que no había nada definido ni reglamentaria ni políticamente, el plebiscito fue una oportunidad para poner a prueba la capacidad organizativa y legal de este órgano electoral ${ }^{9}$.

Dentro de los aspectos más positivos, que pueden ser señalados, se encuentran la apertura puntual de las mesas de votación, la comunicación y relación asertiva con todos los actores involucrados, la detallada atención a las personas que ejercían el voto asistido y la disponibilidad para brindar información a los distintos observadores. Los representantes de las agrupaciones de cada distrito manifestaron confianza y tranquilidad por el hecho de que eran personas funcionarias del TSE quienes se encontraban en las mesas.

El proceso transcurrió con normalidad y de forma ordenada. Los altos porcentajes de participación ciudadana no implicaron incidentes o anomalías durante las votaciones y esto lo constataron los representantes del Cuerpo $\mathrm{Na}$ cional de Delegados, los fiscales acreditados por los partidos políticos Acción Ciudadana, Liberación Nacional, Republicano Socialcristiano y la Unidad Social Cristiana, así como el equipo de observación de las universidades estatales.

Algunas incidencias mínimas detectadas fueron que algunas papeletas estaban mal dobladas y no se podían ver fácilmente las firmas de los miembros de mesa; asimismo, que algunos firmaban las papeletas por adelantado sin existir flujo de votantes; también, se señaló la salida frecuente del aula por parte de integrantes de mesa, sin claridad respecto al proceso seguido para 
registrar su salida y reingreso y que, en algunas juntas receptoras, los pormenores o las incidencias del proceso (votos asistidos, entradas y salidas, cambios de los miembros o fiscales) tampoco fueron debidamente anotados en el acta. Sin embargo, ninguna de estas incidencias afectó el resultado y la transparencia del proceso.

En algunos de los centros de votación no se permitió la presencia de menores de edad al acompañar, sobre todo, a sus madres a la hora de votar, por considerarla incorrecta, porque "podía poner en riesgo el carácter secreto del voto".

\section{Conclusiones}

Las experiencias obtenidas de la participación en distintos procesos electorales han permitido al Centro Agenda Joven de la UNED desarrollar y sistematizar un conocimiento más profundo acerca de los elementos y los factores que caracterizan el comportamiento político electoral de las personas jóvenes en Costa Rica.

Los procesos de consulta popular son relevantes en toda democracia, porque son las propias personas ciudadanas quienes deciden de manera libre y directa, lo que consideran mejor para sus intereses y la comunidad. Como mecanismo de democracia participativa, el plebiscito brinda a la ciudadanía la oportunidad de expresar libremente su opinión y ser parte de la toma de decisiones; la participación en igualdad de condiciones para toda la ciudadanía le otorga la necesaria legitimidad al proceso y fortalece el sistema democrático.

Se determinó en el componente cuantitativo de la investigación que más del $70 \%$ de las personas jóvenes electoras perciben la política con compromiso, entusiasmo y pasión al relegar la indiferencia y el disgusto hacia actividades electorales. En el contexto de las respuestas brindadas, merece especial atención que la tercera parte del sector poblacional consultado manifestó sentir algo de desconfianza, pero el equipo de investigación constató, en los centros de votación donde se realizó el trabajo de campo, que siempre hubo una actitud respetuosa y de confianza al proceso electoral, por lo que no se puede concluir que la desconfianza esté asociada al plebiscito como práctica directa del sistema democrático nacional.

En lo relacionado con las acciones que realizaban durante los procesos electorales, se refuerzan los resultados anteriormente destacados, sobre todo el compromiso y el entusiasmo, puesto que la percepción de la forma en que se involucran y los hallazgos observados por el equipo de investigación comprobaron el ejercicio de la población joven de sus derechos políticos, tanto en aspectos logísticos, como de coordinación y fiscalización.

Aunque el interés por participar en el plebiscito mostró diferencias por sexo, el equipo observador registró que, en el escenario propio de las juntas receptoras de votos, acudieron con la misma responsabilidad y entusiasmo. 
La complementariedad metodológica permitió registrar el fenómeno tal y como es vivido por las personas que habitan las comunidades en que se desarrolló la investigación. La posibilidad de presenciar el proceso en los diferentes momentos de su desarrollo resultó enriquecedor para la comprensión del fenómeno investigado. En particular, el equipo observador registró diversas manifestaciones acerca de la responsabilidad que debían asumir las personas jóvenes en un momento histórico como este, en el que iniciaban el camino hacia la construcción de una identidad cantonal.

Además de la ayuda logística, encontraron, en los medios electrónicos y las redes sociales, herramientas de gran valía para asumir cuotas de responsabilidad y liderazgo en diversas actividades del proceso.

A pesar del entusiasmo mayoritario en las personas jóvenes, otros sectores etarios (sobre todo personas adultas y adultas mayores) no asimilaron de ese mismo modo el plebiscito. Algunas de esas personas lo concibieron como un problema de vacío legal y otras como una discusión producto de conflictos históricos entre habitantes de la zona. Ninguno de estos argumentos fue un impedimento para ejercer el voto, lo cual se evidencia en los altos porcentajes de participación ciudadana, que se suscitaron en un proceso que transcurrió de manera ordenada y sin anomalías durante su desarrollo.

En definitiva, la utilidad de revisar y evaluar las prácticas políticas de las personas jóvenes coloca a las universidades estatales, y a la UNED en particular, en una posición de gran responsabilidad para dar a conocer los intereses, las opiniones y las actitudes de las personas jóvenes hacia la democracia y el sistema político costarricense.

Como posibilidades futuras de investigación, se enfatiza en la necesidad de identificar las causas del abstencionismo en procesos electorales cantonales, así como la utilización de las redes sociales en la divulgación de actividades comunales de toma de decisión y cuál es el papel de las personas jóvenes en el manejo de la información y de los canales para difundirla.

\section{Bibliografía}

Alvarado Chaves, Gustavo. 2014. «Democracia e institucionalidad: La visión de la juventud». Revista del Consejo de la Persona Joven 1(1): 102110.

Asamblea Legislativa de la República de Costa Rica. (1998). Código Municipal Ley $\mathrm{N}^{\circ} 7794$ y sus reformas. Disponible en https://www.tse.go.cr/pdf/normativa/codigomunicipal.pdf

Gibbs, Graham. 2012. El análisis de datos cualitativos en Investigación Cualitativa. Madrid: Ediciones Morata. 
González, Roy. 2017. «Revisión sistemática de literatura sobre comportamiento político y juventud en Latinoamérica y Costa Rica desde 1990 hasta 2016». Revista Rupturas, 7(2): 93-143.

Grajales, Tevni. 2014. Tipos de investigación científica (Puebla, México, 2014), acceso el 10 de febrero de 2019, http://www.iupuebla.com/Maestrias/M_E_GENERO/MA_Maestria_Gene ro/Jose_Miguel_Velez/Tipos\%20de\%20investigacion.pdf

Instituto Nacional de Estadística y Censos (2011). Censo de Población y Vivienda. Disponible en http://www.inec.go.cr/censos/censos-2011

Página de Facebook del Tribunal Supremo de Elecciones, acceso el 26 de marzo 2020, https://www.facebook.com/TSECostaRica/videos/550822358777938

Página de Facebook del Tribunal Supremo de Elecciones, acceso el 26 de marzo 2020, https://www.facebook.com/TSECostaRica/videos/234594347491784

Programa Agenda Joven. 2019. Informe de Observación Plebiscito Río Cuarto, https://investiga.uned.ac.cr/agendajoven/

Taylor, Steve y Bogdan, Robert. 1987. Introducción a los métodos cualitativos de investigación: La búsqueda de significados. Barcelona: Paidós.

Tribunal Supremo de Elecciones. 2019. Decreto de convocatoria al Plebiscito del cantón Río Cuarto, Provincia Alajuela, 02-2019, acceso el 18 de marzo de 2019, http://www.tse.go.cr/pdf/varios/Decreto-2-2019.pdf

Tribunal Supremo de Elecciones. Decreto 03-98. Manual para la realización de consultas populares a escala cantonal y distrital (San José, Costa Rica, 1998), acceso el 4 de abril de 2019, https://www.tse.go.cr/pdf/normativa/consultaspopulares.pdf

Tribunal Supremo de Elecciones. 2009. Reglamento para la observación de procesos electivos y consultivos, acceso el 4 de abril de 2019, http://www.tse.go.cr/pdf/normativa/observacionprocesoselectivos.pdf

Tribunal Supremo de Elecciones. 2019. Resultados oficiales del plebiscito. Resolución N. ${ }^{\circ} 2688-E 11-2019$ del 23 de abril de 2019, acceso el 26 de abril de 2019, https://www.tse.go.cr/juris/relevantes/2688-E11-2019.html 